\title{
A New Approach for Severity Estimation of Transversal Cracks in Multi-layered Beams
}

Abstract

Nowadays, the damage severity evaluation in mechanical structures is mostly performed by analyzing the natural frequency shift. The non-isotropic materials, as the multi-layered ones, are widespread in industrial applications, due to their interesting physicmechanical properties. Thus, a deeper approach of multi-layered beams becomes an important request in the research domain. This paper introduces a damage severity estimator by expressing the crack evolution as a function of stored energy. It is well known that the energy stored in a beam without damage is greater than the energy of that damaged beam. As a consequence, the beam deflection can be related to the stored energy. In this regard, the possibility to split the damage localization and the damage severity assessment has been proven, and also the graphical evolution of the natural frequency shift has been achieved as a function of the crack depth. The results achieved by the finite element method (FEM) and experimental tests are given in tables and graphics. For the first five vibration modes, a comparison was made between frequencies accomplished by analytical, numerical and experimental analyses, in order to give more credibility to the accuracy of the research data presented in this paper.

\section{Keywords}

Multi-layered beam, Damage localization, Damage severity, Stored energy, Natural frequency shift, Vibration-based damage detection
Gilbert-Rainer Gillich ${ }^{\text {a }}$

Nuno M. M. Maia ${ }^{b}$

Ion-Cornel Mituletu ${ }^{a}$

Marius Tufoi ${ }^{\text {a }}$

Vasile lancu $^{\text {a }}$

Zoltan Korka ${ }^{a}$

${ }^{\text {a }}$ Department of Mechanical Engineering

"Eftimie Murgu" University of Resita

320085 Resita, Romania

gr.gillich@uem.ro,mituic@yahoo.com, m.tufoi@uem.ro,v.iancu@uem.ro,

z.korka@uem.ro

${ }^{\mathrm{b}}$ LAETA, IDMEC, Instituto Superior

Tecnico, Univ. de Lisboa, Av. Rovisco

Pais, 1049-001 Lisboa, Portugal. nma-

ia@dem.ist.utl.pt

http://dx.doi.org/10.1590/1679-78252541

Received 15.10.2015

In revised form 19.03.2016

Accepted 28.03.2016

Available online 12.04.2016

\section{INTRODUCTION}

In the last decades, the techniques to monitor structures and evaluate their integrity has been permanently developed and improved. The main idea, on which these techniques are based, is to correlate the dynamical features of structures with their healthy state. In this way, the modal parameter 
changes can indicate the occurrence of damage in the structure and, in some conditions, asses the damage location and severity (Morassi and Vestroni, 2008), (Doebling et al., 1998) and (Gillich et al., 2009). The structural health monitoring effectiveness depends on the applied techniques and the nature of the materials composing the structure (Gillich et al., 2010).

A type of materials more and more used in various engineering applications is the multi-layered composite, due to its characteristics, which can be easily tailored for specific applications. The methods involved to assess damages in that kind of structures depend on the damage type, being mainly delamination and transverse cracks. In the case of cracks, it is important to have a benchmark of their extent. Concerning those structures and as far as the authors are aware, no mathematical relation predicting the frequency change in relation to the crack depth was reported in the literature.

The aim of this paper is to introduce a relation indicating the frequency shift of the bending vibration modes with respect to the damage severity in case of a 5-layered composite. This relation offers an overview on the physical phenomenon, making it understandable, and it is a precious tool in evaluating damage severity in that type of composites.

\section{THE FRACTURE MECHANICS APPROACH}

The following alternative method is performed by many scientists, concerning the model of cracked beams, and it is based on the modeling of damage through a rotational spring (Chondros et al., 1998). The damage is characterized by the spring constant $K_{e q}$, which is expressed in a way to allow an analogy between different empirical expressions of stress intensity factors:

$$
K_{e q}=\frac{1}{s}, \text { with } s=\left(\frac{h}{E I}\right) P(\hat{a})
$$

where $s$ is the damage severity, $P(\hat{a})$ is the expression of the stress intensity factor from fracture mechanics, $E I$ is the healthy beam flexural stiffness, $h$ is the thickness, $a$ is the damage depth and $\hat{a}=a / h$ is the dimensionless damage depth. For the undamaged beam $a=0$ and $K_{e q}=\infty$.

After (Liebowitz et al., 1967), (Rizos et al., 1990), (Okamura et al., 1969) and (Caddemi and Calio, 2009) one has the local compliance given by:

$$
\begin{aligned}
P(\hat{a}) & =5.346\left[1.8624(\hat{a})^{2}-3.95(\hat{a})^{3}+16.375(\hat{a})^{4}-37.226(\hat{a})^{5}+\right. \\
& \left.+76.81(\hat{a})^{6}-126.9(\hat{a})^{7}+172(\hat{a})^{8}-143.97(\hat{a})^{9}+66.56(\hat{a})^{10}\right]
\end{aligned}
$$

Alternatively, (Ostachowicz and Krawczuk, 1991) proposed the following expression:

$$
\begin{aligned}
P(\hat{a})=6 & \pi(\hat{a})^{2}\left[0.6384-1.035(\hat{a})+3.7201(\hat{a})^{2}-5.1773(\hat{a})^{3}+7.553(\hat{a})^{4}\right. \\
& \left.-7.332(\hat{a})^{5}+2.4909(\hat{a})^{6}\right]
\end{aligned}
$$

Imposing a sequential rotation, the models of damage sustained by a continuous description of the beam stiffness reduction in the proximity of damage can be roughly appreciated by a method 
that is fully-flexible, due to the fact that the concentrated flexibility reproduces the relative rotation of cross-section governed by the damage (Gillich et al., 2014).

Some relations of the local bending are offered by (Bilello, 2001):

$$
P(\hat{a})=\frac{(\hat{a})(2-\hat{a})}{0.9(\hat{a}-1)^{2}}
$$

and by (Chondros et al., 1998), who have created a lumped cracked flexibility model equivalent to their continuous model:

$$
\begin{gathered}
P(\hat{a})=6 \pi\left(1-v^{2}\right)\left[0.6272(\hat{a})^{2}-1.04533(\hat{a})^{3}+4.5948(\hat{a})^{4}-9.9736(\hat{a})^{5}+\right. \\
\left.+20.2948(\hat{a})^{6}-33.0351(\hat{a})^{7}+47.1063(\hat{a})^{8}-40.7556(\hat{a})^{9}+19.6(\hat{a})^{10}\right]
\end{gathered}
$$

where $v=0.3$ is the Poisson's ratio.

Fig. 1 presents the expressions of function $P$ in respect to depth $\hat{a}$, where the continuous thick line represents the expression given in relation (2), the dotted line expression (3), the thin line relation (4) and the dashed line relation (5).

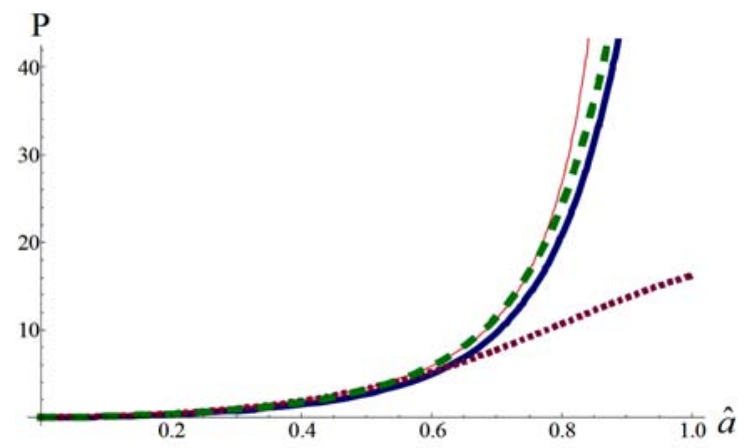

Figure 1: Graphical representation of function $\mathrm{P}$ proposed by different authors.

The previous formulae are empirically derived for rectangular cross-sections. For other crosssection shapes further experimental research is required.

\section{AN EXACT MATHEMATICAL RELATION TO PREDICT FREQUENCY CHANGES IN DAMAGED}

\section{BEAMS}

According to the weak points above highlighted, several conclusions regarding the conditions imposed for the breathing crack models can be formulated. The most important are:

- for each vibration mode, a beam stores an amount of energy dependent on the structural stiffness; hence, one single frequency dependent of the stored energy is expected;

- for the vibration analysis of beams with breathing cracks, the beam stiffness derived from static analysis is inappropriate; 
- the two half periods $\left(T_{o} / 2\right)$, for the open and for the close stages, are identical.

Because the statically derived flexural stiffness EI about the axis of interest is not relevant to characterize the bending vibration of beams with breathing cracks, a new measure is required. According to the heretofore research performed, the deflection increase due to damage, achieved by applying an external load, is proposed as measure of the damage severity.

In Fig. 2, the deflection of the original beam in healthy state due to dead weight is plotted by blue line and the deflection of the same beam having a crack near the fixed end is represented in magenta. Also, an equivalent beam having a reduced uniform flexural rigidity $(E I)^{e q}$ is shown in violet. We assumed both beams as having the same cross-section and mass. Further, the original damaged beam and the equivalent one have the same deflection at the free end, they storing the same amount of energy. This is in accordance to Castigliano's second theorem, about displacements in a linearly elastic structure, which states that if two beams subjected to the same load present similar deflection, then they store the same amount of energy.

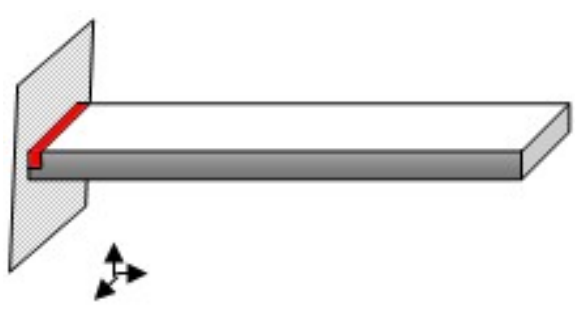

a.

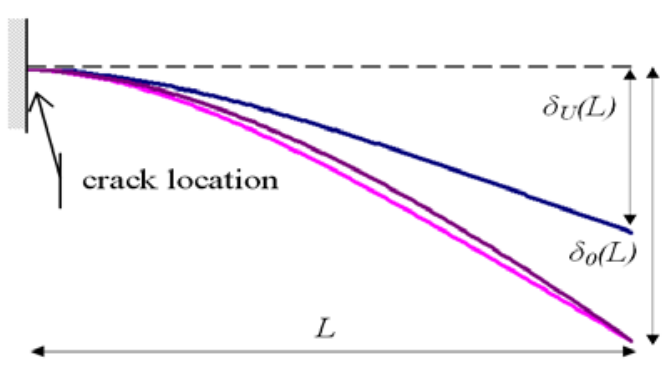

b.

Figure 2: 3D-schematics of the beam with the damage is pointed by red line (a); deflections for the healthy beam, damaged beam in open stage and equivalent beam under the dead mass (b).

For the original healthy cantilever beam, the dead mass produces a deflection $\delta_{U}(L)$ that is derived using the well-known relation:

$$
\delta_{U}(L)=\frac{\rho g A L^{4}}{8 E I}=\frac{M g L^{3}}{8 E I}
$$

where $\rho$ is the mass density, $g$ the gravitational acceleration, $A$ the beam cross-section area, $L$ the length and $M$ the mass. If damage occurs at the fixed end of the original beam (blue), the deflection increases (magenta) to $\delta_{0}(L)$. It happens because of a supplementary rotation in the damaged region, for the rest of the beam the deflection and rotation gradient remaining as in the undamaged case. Now, it can be assumed that a healthy equivalent beam (violet) with a reduced constant rigidity $(E I)^{e q}$ has the same deflection $\delta_{0}(L)$. Note that, the equivalent beam cross-section area and mass density are maintained unchanged, in order to achieve the same mass distribution and total weight as for the original beam. In this case, the deflection of the equivalent beam is: 


$$
\delta_{0}(L)=\frac{\rho g A L^{4}}{8(E I)^{e q}}=\frac{M g L^{3}}{8(E I)^{e q}}
$$

From Eqs. (6) and (7) result the original and equivalent beam rigidities:

$$
E I=\frac{M g L^{3}}{8 \delta_{U}(L)},(E I)^{e q}=\frac{M g L^{3}}{8 \delta_{0}(L)}
$$

It is possible to define the equivalent beam's rigidity, in respect to the original rigidity and the deflection achieved by the two beams at the free end, as being:

$$
(E I)^{e q}=\frac{\delta_{U}(L)}{\delta_{0}(L)} E I
$$

Now, the beam having the weak axis in vertical position is considered. As a consequence, the beam becomes straight, while the bending state under the dead mass is no longer present. By moving the free end away from the rest position with a small distance $w(L)$, the beam stores energy. Releasing the free end, the beam starts to vibrate. In the absence of damping the energy is conserved over time. The strain energy of the original beam achieves the highest value at the extreme positions, which is:

$$
E_{P-i}^{U}=\frac{E I}{2} \int_{0}^{L}\left(w_{i}^{\prime \prime}(x)\right)^{2} d x
$$

This rule is also valid for the damaged beam, which has the maximum strain energy:

$$
E_{P-i}^{0}=\frac{(E I)^{e q}}{2} \int_{0}^{L}\left(w_{i}^{\prime \prime}(x)\right)^{2} d x
$$

Obviously, the healthy beam stores a bigger amount of energy related to the damaged one. At the rest position, the kinetic energy derived for the original beam achieves the maximum, being:

$$
E_{K-i}^{U}=\omega_{U}^{2} \frac{M}{2} \int_{0}^{L}\left(w_{i}(x)\right)^{2} d x
$$

For the damaged beam the maximum kinetic energy is:

$$
E_{K-i}^{0}=\omega_{0}^{2} \frac{M}{2} \int_{0}^{L}\left(w_{i}(x)\right)^{2} d x
$$

Because the damaged beam stores lower energy than the original healthy beam, the angular frequencies are in the relation $\omega_{U-i}>\omega_{0-i}$.

In Eq. (10) to (13) $w_{i}(x)$ denotes the $i$-th mode shape and $w_{i}^{\prime \prime}(x)$ is the curvature for the original healthy and equivalent damaged mode $i$. The previously mentioned functions are not time- 
dependent, therefore indicating the amplitudes of the mode shapes and the curvatures. For the beam with one fixed and one free end

$$
\begin{aligned}
& w_{i}(x)=\cosh \alpha_{i} x-\cos \alpha_{i} x-\frac{\cos \alpha_{i} L+\cosh \alpha_{i} L}{\sin \alpha_{i} L+\sinh \alpha_{i} L}\left(\sinh \alpha_{i} x-\sin \alpha_{i} x\right) \\
& w_{i}^{\prime \prime}(x)=\cosh \alpha_{i} x+\cos \alpha_{i} x-\frac{\cos \alpha_{i} L+\cosh \alpha_{i} L}{\sin \alpha_{i} L+\sinh \alpha_{i} L}\left(\sinh \alpha_{i} x+\sin \alpha_{i} x\right)
\end{aligned}
$$

where $\alpha_{i}$ is the wave number for the beam resulted as solutions of the characteristic equation.

Using Rayleigh's principle, the energies are related as $E_{P-i}^{U}=E_{K-i}^{U}$ respectively $E_{P-i}^{0}=E_{K-i}^{0}$, and the angular frequencies result from Eq. (10) to (13), in:

$$
\begin{aligned}
& \omega_{U-i}^{2}=\frac{\frac{E I}{2} \int_{0}^{L}\left(w_{i}^{\prime \prime}(x)\right)^{2} d x}{\frac{M}{2} \int_{0}^{L}\left(w_{i}(x)\right)^{2} d x} \\
& \omega_{0-i}^{2}=\frac{\frac{(E I)_{C}^{e q}}{2} \int_{0}^{L}\left(w_{i}^{\prime \prime}(x)\right)^{2} d x}{\frac{M}{2} \int_{0}^{L}\left(w_{i}(x)\right)^{2} d x}=\frac{\frac{E I}{2} \frac{\delta_{U}(L)}{\delta_{C}(L)} \int_{0}^{L}\left(w_{i}^{\prime \prime}(x)\right)^{2} d x}{\frac{M}{2} \int_{0}^{L}\left(w_{i}(x)\right)^{2} d x}
\end{aligned}
$$

From Eqs. (16) and (17), the ratio between the angular frequencies of the healthy beam, for any transverse vibration mode, and the corresponding angular frequencies of the damaged beam is:

$$
\frac{\omega_{0-i}^{2}}{\omega_{U-i}^{2}}=\frac{\frac{E I}{2} \frac{\delta_{U}(L)}{\delta_{C}(L)} \int_{0}^{L}\left(w_{i}^{\prime \prime}(x)\right)^{2} d x}{\frac{M}{2} \int_{0}^{L}\left(w_{i}(x)\right)^{2} d x} \cdot \frac{\frac{M}{2} \int_{0}^{L}\left(w_{i}(x)\right)^{2} d x}{\frac{E I}{2} \int_{0}^{L}\left(w_{i}^{\prime \prime}(x)\right)^{2} d x}=\frac{\delta_{U}(L)}{\delta_{C}(L)}
$$

Thus, the natural frequencies for a beam with stiffness decrease at the fixed end can be calculated by:

$$
f_{0-i}=f_{U-i} \sqrt{\frac{\delta_{U}(L)}{\delta_{0}(L)}}
$$

Note that Eq. (19) can be used for beams with any boundary conditions, because the ratio of the deflections $\delta_{U}(L)$ and $\delta_{0}(L)$ just depends on the healthy and damaged beam stiffness, see Eq. (9). The single difference consists in the location where the damage and the maximum deflection are placed.

Eq. (15) is valid for breathing cracks, i.e. cracks with the two walls in contact at the rest position. Since the frequency drop is just related to the energy decrease, the relation is valid for open cracks as well, but in this case also a frequency increasing due to loss of mass is expected. 
The frequency change $\Delta f_{0-i}=f_{U-i}-f_{0-i}$ is an important indicator in damage detection. It can be derived from Eq. (19), as:

$$
\Delta f_{0-i}=f_{U-i}-f_{0-i}=f_{U-i}\left(1-\sqrt{\frac{\delta_{U}(L)}{\delta_{0}(L)}}\right)=f_{U-i} \frac{\sqrt{\delta_{0}(L)}-\sqrt{\delta_{U}(L)}}{\sqrt{\delta_{0}(L)}}
$$

It is interesting to see how the frequencies change if the damage is not located at the fixed end. For this reason we consider a beam like that presented in Fig. 3, where three areas can be observed: the two healthy segments, having the moment of inertia $I=B H^{3} / 12$, and the damaged slice with $I_{C}=B h^{3} / 12$. In the damaged area the neutral axis is deviated to the cross-section mid.

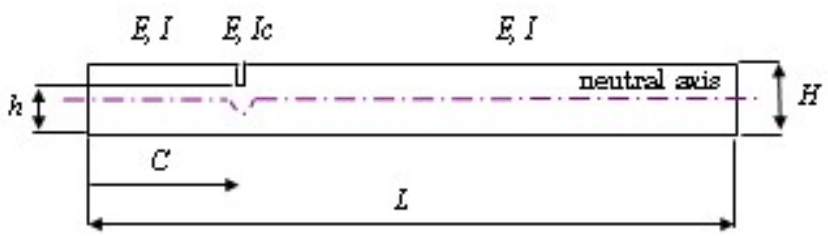

Figure 3: Typical areas for a cracked beam

While the mass distribution still constant, the bending moment $M_{i}(x)$ is easily derived for the healthy cantilever beam, being known that it is proportional to the mode shape second derivative. Thus, the dimensionless bending moment is similar to the dimensionless curvature $\bar{M}_{i}(x) \doteq \bar{w}_{i}^{\prime \prime}(x)$. Here, both terms of the relation are made dimensionless, by the division to the highest value of the category; these are $M_{i}(0)$ and $w_{i}^{\prime \prime}(0)$.

In the case of partial stiffness decrease, as shown in Fig. 3, it is convenient to consider the effective bending moment $M_{\text {eff-i }}(x)$. It is the moment acting on a presumed healthy beam, but producing a similar effect as $M_{i}(x)$ on the damaged one. The relation between the two bending moments is:

$$
M_{e f f-i}(x)=M_{i}(x) \frac{I_{\text {actual }}}{I_{\text {reference }}}
$$

This has as consequences an increasing of the effective bending moment in the damaged slice. In Fig. 4 the apparent energy increase is presented for mode one and three. The blue bars indicate the normalized strain energy distribution along the healthy beam, derived as:

$$
E_{P-i}^{U}=\frac{1}{2 E I} \int_{0}^{L}\left[M_{i}(x)\right]^{2} d x
$$

From Fig. 4 results the participation of each slice to the total stain energy, for the different bending vibration modes. 


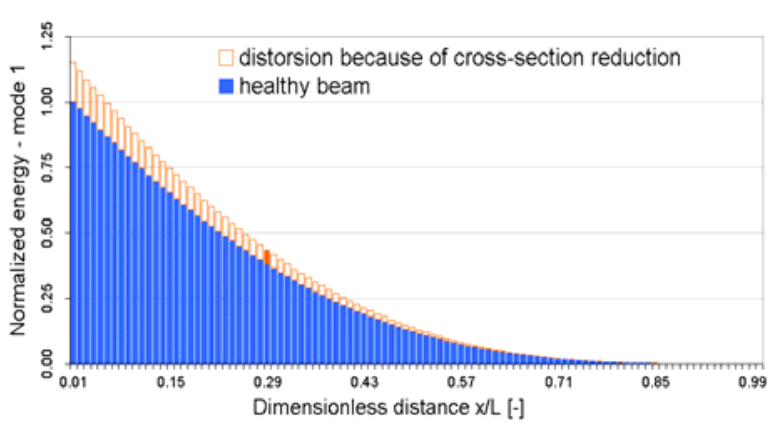

a.

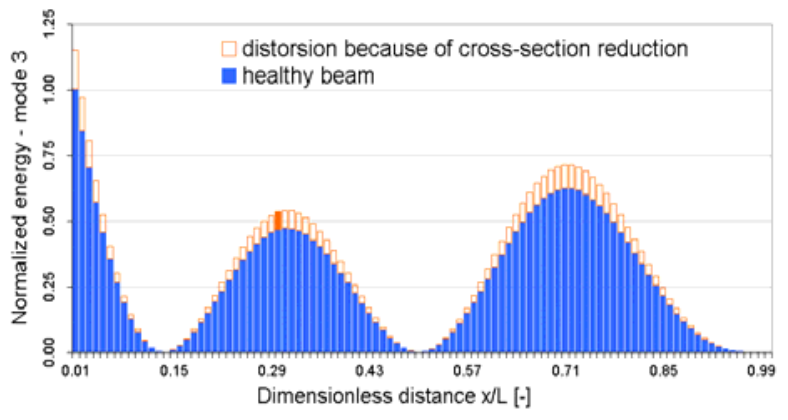

b.

Figure 4: The energy distributions for mode 1 and 3 of a damaged beam.

The bars plotted by orange border reflect the apparent energy increase, if damage occurs in the individual slices. For the slice having as limits the distances $a$ and $b$ measured from the fixed end, the strain energy is:

$$
\left.E_{P-i}^{C}\right|_{a} ^{b}=\frac{1}{2 E I} \int_{a}^{b}\left[M_{e f f-i}(x)\right]^{2} d x=\frac{1}{2 E I_{C}} \int_{a}^{b}\left[M_{i}(x)\right]^{2} d x
$$

The orange full bar shows this increasing for the slice located around distance $x / L=0.29$. Thus, the energy loss ratio is derived from the damaged and healthy state, as:

$$
\eta_{i}=\frac{E_{P-i}^{C}}{E_{P-i}^{U}}=\frac{\frac{1}{2 E I} \int_{0}^{L}\left[M_{e f f-i}(x)\right]^{2} d x}{\frac{1}{2 E I} \int_{0}^{L}\left[M_{i}(x)\right]^{2} d x}
$$

For the damaged case, the energy distribution along the beam in the explicit form is:

$$
E_{P-i}^{C}=\frac{1}{2 E I} \int_{0}^{a}\left[M_{i}(x)\right]^{2} d x+\frac{1}{2 E I_{C}} \int_{a}^{b}\left[M_{i}(x)\right]^{2} d x+\frac{1}{2 E I} \int_{b}^{L}\left[M_{i}(x)\right]^{2} d x
$$

where $0-a$ and $b-L$ are the healthy segments and $a-b$ is the damaged slice.

In agreement with the Eq. (21) and considering the inverse effect of stiffness decrease to the stored energy, we can replace the actual bending moment in Eq. (25) by $M(x)=\left(I_{C} / I\right) M_{C}(x)$. By adding and subtracting the strain energy of the healthy slice $a-b$ in Eq. (25), one attains:

$$
E_{P-i}^{C}=E_{P-i}^{C}+\frac{1}{2 E I_{C}} \int_{a}^{b} \frac{I_{C}^{2}}{I^{2}}\left[M_{i}(x)\right]^{2} d x-\frac{1}{2 E I} \int_{a}^{b}\left[M_{i}(x)\right]^{2} d x
$$

or in a comprehensive form:

$$
E_{P-i}^{C}=E_{P-i}^{C}-\left(1-\frac{I_{C}}{I}\right) \frac{1}{2 E I} \int_{a}^{b}\left[\bar{M}_{i}(x)\right]^{2} d x
$$


Substituting $E_{P-i}^{C}$ in Eq. (22) and reducing the common factor, the energy loss ration becomes:

$$
\eta_{i}=\frac{\int_{0}^{L}\left[M_{i}(x)\right]^{2} d x}{\int_{0}^{L}\left[M_{i}(x)\right]^{2} d x}-\frac{I-I_{C}}{I} \frac{\int_{a}^{b}\left[M_{i}(x)\right]^{2} d x}{\int_{0}^{L}\left[M_{i}(x)\right]^{2} d x}=1-\frac{I-I_{C}}{I} \frac{\int_{a}^{b}\left[M_{i}(x)\right]^{2} d x}{\max \left[M_{i}(x)\right]^{2}} \frac{\max \left[M_{i}(x)\right]^{2}}{\int_{0}^{L}\left[M_{i}(x)\right]^{2} d x}
$$

In Eq. (28) the bending moment is made dimensionless by normalization. Let us denoted this moment as $\bar{M}_{i}(x)$ and the moment acting in slice $a-b$ as $\tau_{i}=\int_{a}^{b}\left[\bar{M}_{i}(x)\right]^{2} d x$. Knowing that $\int_{0}^{L}\left[\bar{M}_{i}(x)\right]^{2} d x=0.25$, the energy loss ratio becomes:

$$
\eta_{i}=1-\frac{4 \tau_{i}\left(I-I_{C}\right)}{I}
$$

From Eq. (22) and Eq. (29), the relation between the natural frequencies for the beam with stiffness decrease and the healthy, it results:

$$
\frac{f_{C-i}}{f_{U-i}}=\sqrt{\frac{E_{P-i}^{C}}{E_{P-i}^{U}}}=\sqrt{\eta_{i}}=\sqrt{1-\frac{4 \tau_{i}\left(I-I_{C}\right)}{I}}
$$

hence, the damaged beam's frequencies are:

$$
f_{C-i}=f_{U-i} \sqrt{1-\frac{4 \tau_{i}\left(I-I_{C}\right)}{I}}
$$

and the frequency decrease due to the crack results:

$$
\Delta f_{C-i}=f_{U-i}-f_{C-i}=f_{U-i}\left[1-\sqrt{1-\frac{4 \tau_{i}\left(I-I_{C}\right)}{I}}\right]
$$

In the following, the frequency decrease is illustrated for a damage iteratively placed along the beam. The stiffness reduction manifests on a slice of width $0.01 \cdot L$, thus one hundred cases are analyzed. Fig. 5 depicts the frequency shift for the vibration modes one and three, all analyzed cases being presented.

Having a look onto Fig. 4 and 5, one can observe that the frequency drop is most important if the crack is located on slices where the normalized bending moment $\bar{M}_{i}(x)$, i.e. the normalized curvature $\bar{w}_{i}^{\prime \prime}(x)=\frac{w_{i}^{\prime \prime}(x)}{\max \left[w_{i}^{\prime \prime}(x)\right]}$, is the unity, thus taking the highest value. For example, it is the fixed end for the cantilever beam, or the both ends of a double-clamped beam. In the case of simply supported beam, this location is reconfigured at each mode, for the first one being the mid-span. 


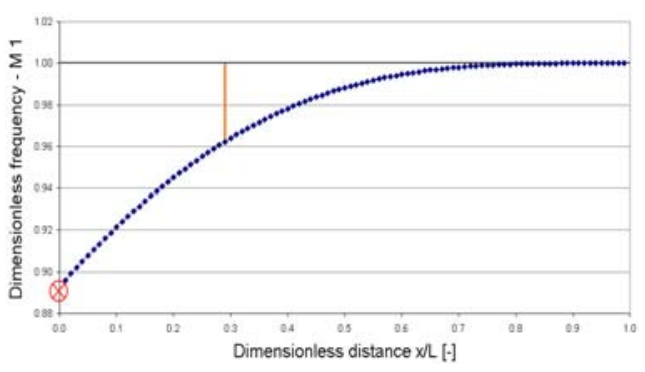

a.

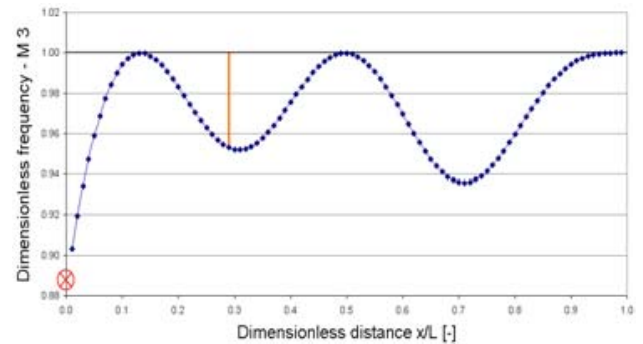

b.

Figure 5: Relative frequency shifts for the stiffness loss on a slice of width 0.01·L.

From Fig. 4 and 5, one can also conclude that for the damage located in slices on which no bending moment acts, thus $\bar{w}_{i}^{\prime \prime}(x)=0$, the frequency decrease is null. In intermediate positions the frequency change depends on the square of the normalized curvature $\left(\bar{w}_{i}^{\prime \prime}(x)\right)^{2}$. Regarding the dimensionless frequency drop achieved for the fixed end, it is similar for all weak-axis bending modes, being around 0.11 in our exemplification (see the red circle). This demonstrates that the severity is one for all modes, the effect being weighted by the square of the normalized curvature $\left(\bar{w}_{i}^{\prime \prime}(x)\right)^{2}$. Therefore, it is convenient to use the frequency decrease for the location with the highest frequency drop to express the damage severity:

$$
\gamma=\frac{\sqrt{\delta_{0}(L)}-\sqrt{\delta_{U}(L)}}{\sqrt{\delta_{0}(L)}}
$$

Consequently, the frequency shift and the relative frequency shift (RFSh) for a damaged EulerBernoulli beam with the transversal crack located at distance $C$ from the fixed end are:

$$
\begin{gathered}
\Delta f_{C-i}=f_{U-i} \frac{\sqrt{\delta_{0}(L)}-\sqrt{\delta_{U}(L)}}{\sqrt{\delta_{0}(L)} \cdot\left(\bar{w}_{i}^{\prime \prime}(C)\right)^{2}} \\
\Delta \bar{f}_{C-i}=\frac{\Delta f_{C-i}}{f_{U-i}}=\frac{\sqrt{\delta_{0}(L)}-\sqrt{\delta_{U}(L)}}{\sqrt{\delta_{0}(L)}} \cdot\left(\bar{w}_{i}^{\prime \prime}(C)\right)^{2}
\end{gathered}
$$

The natural frequency for this beam is

$$
f_{C-i}=f_{U-i}\left[1-\frac{\sqrt{\delta_{C}(L)}-\sqrt{\delta_{U}(L)}}{\sqrt{\delta_{C}(L)}}\right]=f_{U-i}\left[1-\frac{\sqrt{\delta_{0}(L)}-\sqrt{\delta_{U}(L)}}{\sqrt{\delta_{0}(L)}} \cdot\left(\bar{w}_{i}^{\prime \prime}(C)\right)^{2}\right]
$$

This relation can be used to derive the frequency shift due to damage for beams with any support type, any crack location and severity. Moreover, it is applicable to beams with any crosssection shape. The relation can be also used to contrive a database, containing numerous damage scenarios, which constitutes a benchmark for damage detection methods (Gillich and Praisach, 2014), even if composite structures are analyzed (Gillich et al., 2012). 


\section{FINITE ELEMENT ANALISYS OF A 5-LAYER DAMAGED BEAM}

The numerical analysis of the damage depth effect on a 5-layered beam has been performed by means of Finite Element Method (FEM) in the ANSYS simulation software. The sandwich model was structured on three layers of steel and two of PVC, each one having $1 \mathrm{~mm}$ thickness. Steel layers are placed in the exterior and in the middle, PVC layers were interposed between them. We made use of rigid PVC, explicitly Poly (vinyl chloride), because it is a synthetic plastic polymer commonly used in construction.

The most significant material properties for PVC and steel are provided in Table 1. It has to be specified that all the FEM simulations were performed by involving these material properties. Relevant values of the geometrical characteristics for the beam model are presented in Table 2.

\begin{tabular}{cccc}
\hline \hline Name & Poissin's ratio $v$ & Mass density $\rho$ & Young's modulus $E$ \\
\hline Unit & - & $\mathrm{kg} / \mathrm{m}^{3}$ & $\mathrm{~N} / \mathrm{m}^{2}$ \\
PVC & 0.3825 & 1300 & $2.41 \cdot 10^{9}$ \\
Steel & 0.3 & 7850 & $2 \cdot 10^{11}$ \\
\hline \hline
\end{tabular}

Table 1: PVC and steel material properties

\begin{tabular}{ccccc}
\hline \hline Parameter & Length $L$ & Width $B$ & Thickness $h$ & Mass $M$ \\
\hline Unit & $\mathrm{mm}$ & $\mathrm{mm}$ & $\mathrm{mm}$ & $\mathrm{kg}$ \\
Value & 1000 & 50 & 5 & 0.523 \\
\hline \hline
\end{tabular}

Table 2: Geometrical characteristics of 5-layered beam

A static analysis was performed to find the beam deflections under dead mass. After that, the frequencies where investigated. The investigation firstly focused on the healthy beam. Afterwards, the beam cross section was locally reduced at the distance $C=6 \mathrm{~mm}$ from the fixed end by a step of $0.4 \mathrm{~mm}$ up to $4.8 \mathrm{~mm}$. Depth levels around 1 and $3 \mathrm{~mm}$ were additionally considered in order to refine the analysis, in the end a number of 32 damage scenarios was resulted. For all damage scenarios, the damage width is $1 \mathrm{~mm}$ and its length is $50 \mathrm{~mm}$ the same with the beam width (see Fig. 6). The first and the last case are depicted in Fig. 6 .

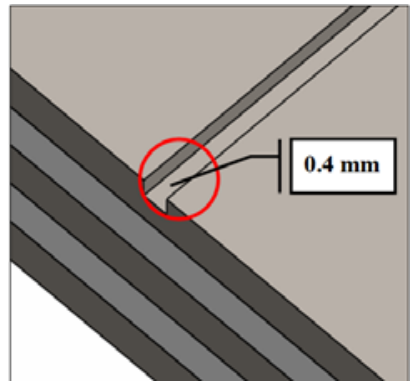

a.

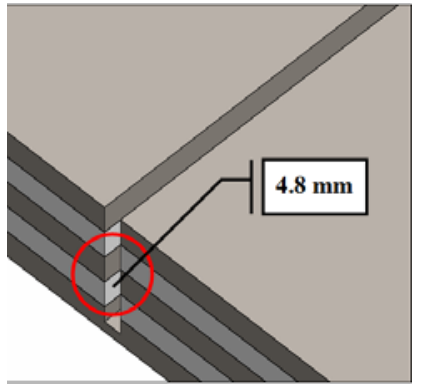

b.

Figure 6: Presence of damage depth by two limit value in the 5-layer beam. 
The simulation of different damage scenarios indicated the way of frequency shift in the case of damage evolution. The achieved natural frequency values are given in Table 3, together with the relative frequency shift $\Delta \bar{f}_{C-i}$ derived with Eq. (36). In parallel, the deflection increase is indicated as well as the damage severity derived from Eq. (33).

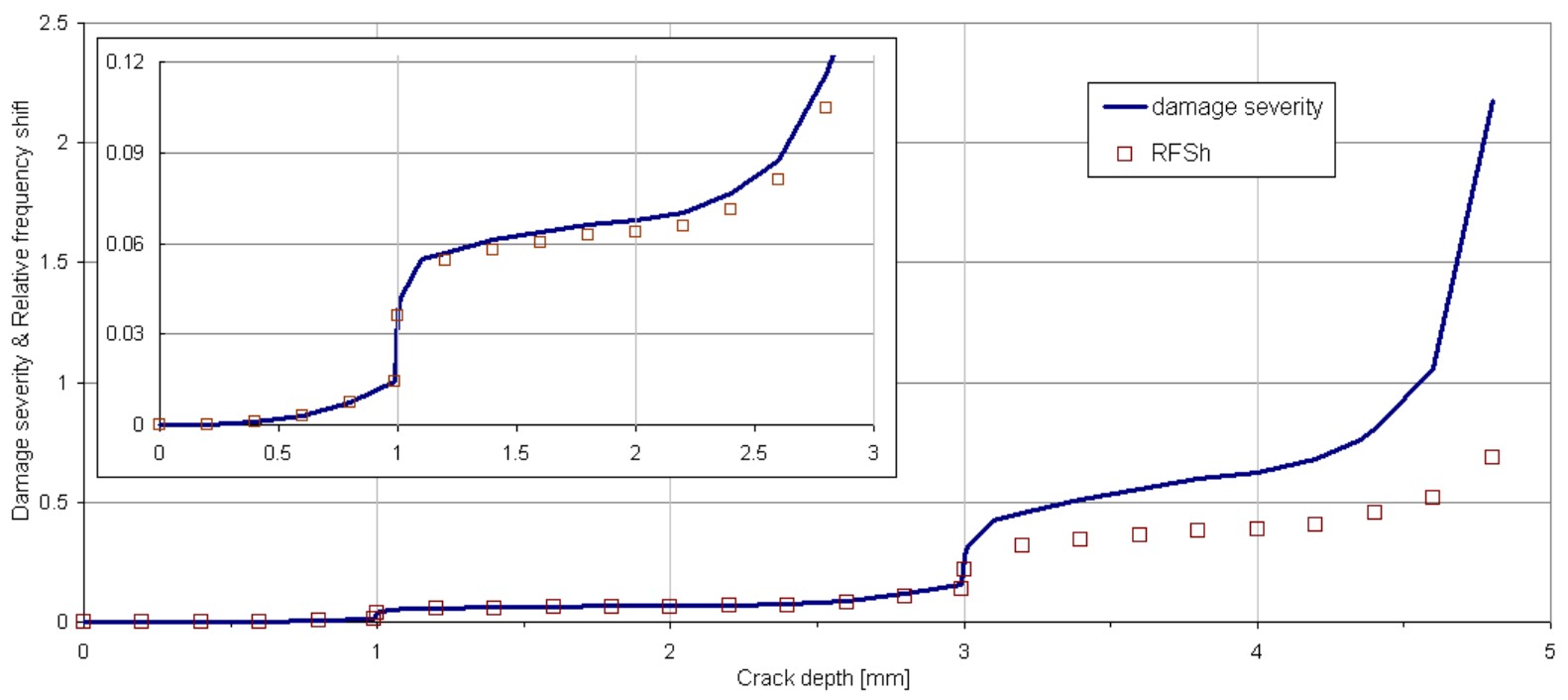

Figure 7: Evolution of damage severity against relative frequency shift.

For the damage depth $0-4.8 \mathrm{~mm}$ a graphical representation of the damage severity evolution obtained by FEM is plotted by blue line in Fig. 7. Also, for the incipient damage depth a zoom of the curve is shown, in order to highlight the small severity values in this depth range. One can observe a sharp severity increase, when the steel sheets are completely sawn; it happens at damage depths of 1 and $3 \mathrm{~mm}$. Thus, for an increased depth, over $3 \mathrm{~mm}$, the reduced beam rigidity in the damaged area favors a strong severity increasing. The severity dramatically advances after the depth of $4 \mathrm{~mm}$ is achieved. It is worth to be mentioned that the plastic behavior occurs for extended depth, which explains the significant increase of deflection. The severity curve is compared against the relative frequency shift evolution derived for the first bending vibration mode. It was found a very good concordance between these two parameters, from the early damage stage until the plastic behavior occurs. This demonstrates that the proposed damage severity indicator is suitable for the damage detection propose.

Tables 4 and 5 present the first ten natural frequencies for a damage located at $274 \mathrm{~mm}$ from the fixed end, considering twelve relevant damage depths. The representation of the relative frequency shift evolution achieved for these modes, is shown in Fig. 8. The normalization is performed in order to assure a qualitative comparison of these evolution curves by involving Eq. (36). 


\begin{tabular}{|c|c|c|c|c|}
\hline $\begin{array}{c}\text { Damage depth } \\
{[\mathrm{mm}]}\end{array}$ & $\begin{array}{c}\text { Frequency } \\
{[\mathrm{Hz}]}\end{array}$ & $\begin{array}{c}\text { Relative frequen- } \\
\text { cy shift [-] }\end{array}$ & $\begin{array}{c}\text { Deflection } \\
{[\mathrm{mm}]}\end{array}$ & $\begin{array}{c}\text { Damage severity } \\
{[-]} \\
\end{array}$ \\
\hline 0 & 4.457588 & 0 & 19.31522 & 0 \\
\hline 0.2 & 4.456568 & 0.0002288 & 19.32448 & 0.000239 \\
\hline 0.4 & 4.452995 & 0.0010304 & 19.35555 & 0.001043 \\
\hline 0.6 & 4.445269 & 0.0027636 & 19.42275 & 0.00278 \\
\hline 0.8 & 4.423971 & 0.0075415 & 19.60975 & 0.007595 \\
\hline 0.99 & 4.39469 & 0.0141103 & 19.87135 & 0.014294 \\
\hline 1 & 4.297331 & 0.0359516 & 20.77851 & 0.037188 \\
\hline 1.01 & - & - & 20.97943 & 0.04219 \\
\hline 1.1 & - & - & 21.48587 & 0.054694 \\
\hline 1.2 & 4.216425 & 0.0541017 & 21.57843 & 0.056964 \\
\hline 1.4 & 4.199927 & 0.0578029 & 21.7475 & 0.061096 \\
\hline 1.6 & 4.189053 & 0.0602421 & 21.85939 & 0.063822 \\
\hline 1.8 & 4.178995 & 0.0624987 & 21.9639 & 0.066363 \\
\hline 2 & 4.174335 & 0.0635441 & 22.01288 & 0.067551 \\
\hline 2.2 & 4.164286 & 0.0657985 & 22.11845 & 0.070108 \\
\hline 2.4 & 4.139949 & 0.071258 & 22.37691 & 0.076342 \\
\hline 2.6 & 4.097167 & 0.0808555 & 22.84288 & 0.087491 \\
\hline 2.8 & 3.991261 & 0.1046142 & 24.05981 & 0.116082 \\
\hline 2.99 & 3.857027 & 0.1347278 & 25.74397 & 0.154484 \\
\hline 3 & 3.489578 & 0.21716 & 31.36642 & 0.274332 \\
\hline 3.01 & - & - & 33.25827 & 0.3122 \\
\hline 3.1 & - & - & 39.26845 & 0.425844 \\
\hline 3.2 & 3.053069 & 0.3150849 & 40.81481 & 0.453647 \\
\hline 3.4 & 2.936541 & 0.3412265 & 44.06856 & 0.510479 \\
\hline 3.6 & 2.854865 & 0.3595493 & 46.58771 & 0.553051 \\
\hline 3.8 & 2.770193 & 0.3785444 & 49.43836 & 0.599861 \\
\hline 4 & 2.727175 & 0.3881949 & 50.98671 & 0.62472 \\
\hline 4.2 & 2.64152 & 0.4074106 & 54.30344 & 0.676733 \\
\hline 4.35 & - & - & 59.66686 & 0.757587 \\
\hline 4.4 & 2.438428 & 0.4529715 & 63.59422 & 0.814508 \\
\hline 4.6 & 2.146669 & 0.5184237 & 81.81808 & 1.058139 \\
\hline 4.8 & 1.386807 & 0.6888884 & 194.7711 & 2.175502 \\
\hline
\end{tabular}

Table 3: Dimensionless frequency shifts for the first vibration mode and the deflections under the own mass considering the damage depth between 0 and $4.8 \mathrm{~mm}$ 


\begin{tabular}{ccccccc}
\hline \hline \multirow{2}{*}{$\begin{array}{c}\text { Mode } \\
\text { number } i\end{array}$} & \multicolumn{5}{c}{ Damage depth $[\mathrm{mm}]-$ Damage location $C=274[\mathrm{~mm}]$} \\
\cline { 2 - 6 } & 0 & 0.4 & 0.8 & 1.2 & 1.6 & 2 \\
\hline 1 & 4.457588 & 4.453703 & 4.44109 & 4.323778 & 4.309279 & 4.300839 \\
2 & 27.84403 & 27.84233 & 27.83379 & 27.74211 & 27.73108 & 27.7247 \\
3 & 77.56287 & 77.50425 & 77.29646 & 75.37508 & 75.15085 & 75.0222 \\
4 & 150.8723 & 150.7908 & 150.5094 & 148.0918 & 147.8249 & 147.6733 \\
5 & 247.0677 & 247.0654 & 247.0647 & 247.0583 & 247.0544 & 247.0511 \\
6 & 364.9251 & 364.7244 & 364.0628 & 358.1151 & 357.4334 & 357.0411 \\
7 & 503.067 & 502.5984 & 501.0597 & 488.8718 & 487.629 & 486.9366 \\
8 & 659.9959 & 659.8149 & 659.2557 & 655.0976 & 654.6692 & 654.4304 \\
9 & 834.1504 & 834.0533 & 833.8118 & 831.6734 & 831.41 & 831.2426 \\
10 & 1023.954 & 1023.159 & 1020.622 & 999.0905 & 996.7881 & 995.495 \\
\hline \hline
\end{tabular}

Table 4: Frequency values for ten vibration modes considering the damage depth between 0 and $2 \mathrm{~mm}$

\begin{tabular}{ccccccc}
\hline \hline \multirow{2}{*}{$\begin{array}{c}\text { Mode } \\
\text { number } i\end{array}$} & \multicolumn{6}{c}{ Damage depth $[\mathrm{mm}]-$ Damage location $C=274[\mathrm{~mm}]$} \\
\cline { 2 - 7 } 1 & 2.4 & 2.8 & 3.2 & 3.6 & 4 & 4.4 \\
\hline 2 & 4.270118 & 4.203756 & 3.563213 & 3.419569 & 3.318866 & 2.805408 \\
3 & 27.70243 & 27.65384 & 27.19954 & 27.10738 & 27.0445 & 26.75454 \\
4 & 74.56347 & 73.60228 & 66.35173 & 65.15142 & 64.38062 & 61.19796 \\
5 & 147.1425 & 146.0627 & 139.4981 & 138.6129 & 138.0717 & 136.0146 \\
6 & 247.0491 & 247.0436 & 247.0193 & 247.0075 & 246.994 & 246.9909 \\
7 & 355.6458 & 352.8154 & 333.9272 & 331.3033 & 329.6788 & 323.4274 \\
8 & 484.5077 & 479.8296 & 457.4701 & 455.1791 & 453.8575 & 449.0691 \\
9 & 653.6296 & 652.0409 & 644.5765 & 643.7273 & 643.2199 & 641.3758 \\
10 & 830.7425 & 829.7737 & 822.2759 & 821.0884 & 820.2481 & 816.9608 \\
\hline \hline
\end{tabular}

Table 5: Frequency values for ten vibration modes considering the damage depth between 0 and $4.8 \mathrm{~mm}$

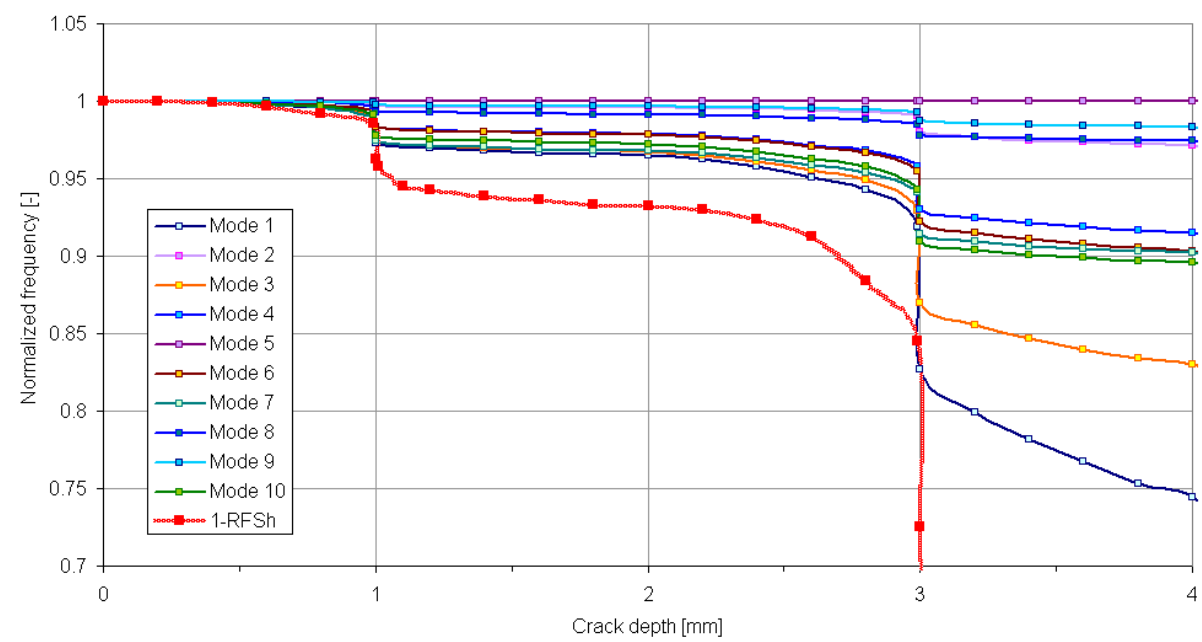

Figure 8: Normalized frequency shift evolution of a 5-layered beam, taken for the relevant domain of analysis 
By representing the frequency shift as the effect of damage depth evolution, it was verified that it takes the same allure for all vibration modes. This demonstrates the fact that the split of damage location issue and damage severity evaluation is very possible.

From Fig. 8 it is quite easy to observe that the allure of the normalized frequencies is similar to the damage severity, being in the relation:

$$
\bar{f}_{C-i}=\frac{f_{C-i}}{f_{U-i}}=1-\frac{\sqrt{\delta_{0}(L)}-\sqrt{\delta_{U}(L)}}{\sqrt{\delta_{0}(L)}} \cdot\left(\bar{w}_{i}^{\prime \prime}(C)\right)^{2}=1-\gamma \cdot\left(\bar{w}_{i}^{\prime \prime}(C)\right)^{2}
$$

In fact, it is easy to accomplish the normalized frequency of any bending mode for the cracked beam from the damage severity $\gamma$ and the square of the curvature $\left(\bar{w}_{i}^{\prime \prime}(C)\right)^{2}$. At the mode five, which has an inflection point at $C=274 \mathrm{~mm}$, the frequency shift is minimal. In contrast, mode one achieves the highest curvature among all the ten analyzed modes, thus the biggest frequency change is observed.

\section{EXPERIMENTAL TESTS ON A 5-LAYER DAMAGED BEAM}

Experimental tests have been performed on a beam of 5 layers. The beam structure is like the one described in section 3 and also the distance of damage accomplishment is $C=274 \mathrm{~mm}$. The test stand, presented in Fig. 9.a, is structured to meet real condition and also those from numerical simulation. Obviously, the goal was to confirm the method results achieved by mathematical calculus and FEM.

To complete the mechanical part of the stand, a vibration data processing and analyzing system was provided. The system is based on a piezo-accelerometer Kistler 8772, designed to fine sensing the vibration waves and send three analog signals, corresponding to each axis, to the acquisition board. Acquisition board consists of a compact chassis NI cDAQ-9172 and the signal acquisition module NI 9234. Acquisition board sends binary encoding data to the computer. The computer runs an application in LabView software, special designed to fine processing the vibration data, for accurate frequency identification (Onchis et al., 2012). The accelerometer physical position is nearby the free end of the beam. A general view of the test stand is given in Fig. 9.a. The accelerometer position (SP) is highlighted.

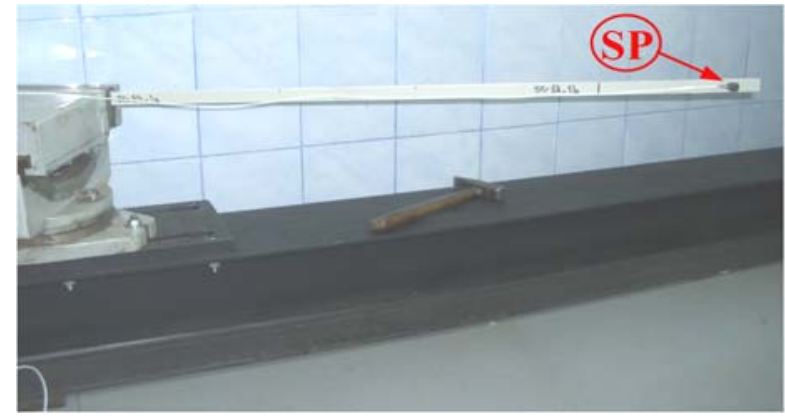

a)

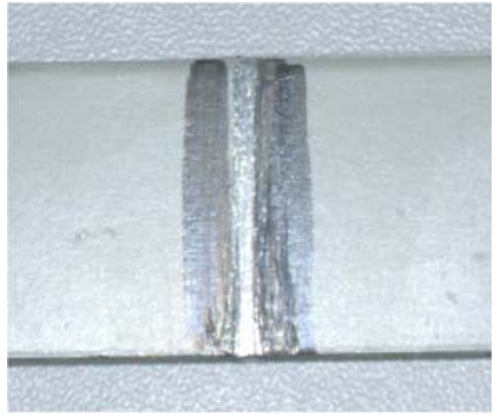

b)

Figure 9: General view of the test stand and magnification of the first step damage 
Crack evolution was achieved by a $0.4 \mathrm{~mm}$ sequential step saw cutting, until the first layer of steel was entirely damaged and the second layer of PVC was almost damaged. In the last step, the damage reached a $1.6 \mathrm{~mm}$ depth. This strategy has been adopted because the last $0.4 \mathrm{~mm}$ of PVC does not significantly affect the entire beam stiffness.

For each damage step, a number of five tests in identical testing conditions have been undertaken. Each value shown in Tables 6 to 8 represents the average of five measurements. Fig. 9.b depicts the first damage step, the breathing space being bordering by black areas for more clearness.

To have a better visibility over the results, Table 6 shows an edifying comparison between the frequency values achieved from mathematical calculus (Mat), FEM and average of experimental tests $(\mathrm{Au})$, regarding the first five vibration modes of the undamaged 5-layer beam.

\begin{tabular}{cccc}
\hline \hline \multirow{2}{*}{ Mode } & \multicolumn{3}{c}{ Frequency results $[\mathrm{Hz}]$} \\
\cline { 2 - 4 } & Mat & FEM & $\mathrm{A}_{\mathrm{U}}$ \\
\hline 1 & 4.452 & 4.453 & 4.446 \\
2 & 27.9 & 27.818 & 27.864 \\
3 & 78.128 & 77.502 & 77.803 \\
4 & 153.102 & 150.78 & 151.989 \\
5 & 253.061 & 246.96 & 248.592 \\
\hline \hline
\end{tabular}

Table 6: Measurements of 5-layer healthy beam

Table 7 offers an example of the test results evaluation for the second damage level. The average $\left(A_{D 2}\right)$ of the five test values, for the first five vibration modes, is given. In Table 8, four-figure values have been taken, in order to increase the accuracy of interpretation, so that closer values can be distinguished in an easier way. While the third vibration mode in FEM has an important evolution, in the experimental part it is less visible. As a general observation, in case of higher or lower vibration modes, the evolution cannot be clearly identified, the results tend to spread over randomly.

\begin{tabular}{ccccccc}
\hline \hline \multirow{2}{*}{ Mode } & \multicolumn{5}{c}{ Tests results of frequency measurement $[\mathrm{Hz}]$} & \multirow{2}{*}{ AD2 [Hz] } \\
\cline { 2 - 5 } & 1 & 2 & 3 & 4 & 5 & \\
\hline 1 & 4.406 & 4.416 & 4.418 & 4.424 & 4.419 & 4.417 \\
2 & 27.755 & 27.673 & 27.759 & 27.864 & 28.146 & 27.839 \\
3 & 77.453 & 77.474 & 77.459 & 77.498 & 77.513 & 77.479 \\
4 & 150.842 & 151.648 & 152.023 & 151.973 & 152.125 & 151.722 \\
5 & 247.629 & 247.879 & 248.674 & 249.395 & 248.866 & 248.489 \\
\hline \hline
\end{tabular}

Table 7: Measurements of 5-layer beam, having a $0.8 \mathrm{~mm}$ damage depth and $x=274 \mathrm{~mm}$

\begin{tabular}{cccccc}
\hline \hline \multirow{2}{*}{ Mode } & \multicolumn{5}{c}{ Frequency measurements [Hz] } \\
\cline { 2 - 6 } & 0 & 0.4 & 0.8 & 1.2 & 1.6 \\
\hline 1 & 4.4459 & 4.4376 & 4.4171 & 4.4116 & 4.4112 \\
2 & 27.8641 & 27.8573 & 27.8394 & 27.8312 & 27.8294 \\
3 & 77.8027 & 77.7519 & 77.4788 & 77.4370 & 77.4363 \\
4 & 151.9892 & 151.8990 & 151.7222 & 151.6311 & 151.6294 \\
5 & 248.5915 & 248.5902 & 248.4893 & 248.4440 & 248.4396 \\
\hline \hline
\end{tabular}

Table 8: Frequency measurements of 5-layer beam for damage evolution from 0.4 to 1.6 


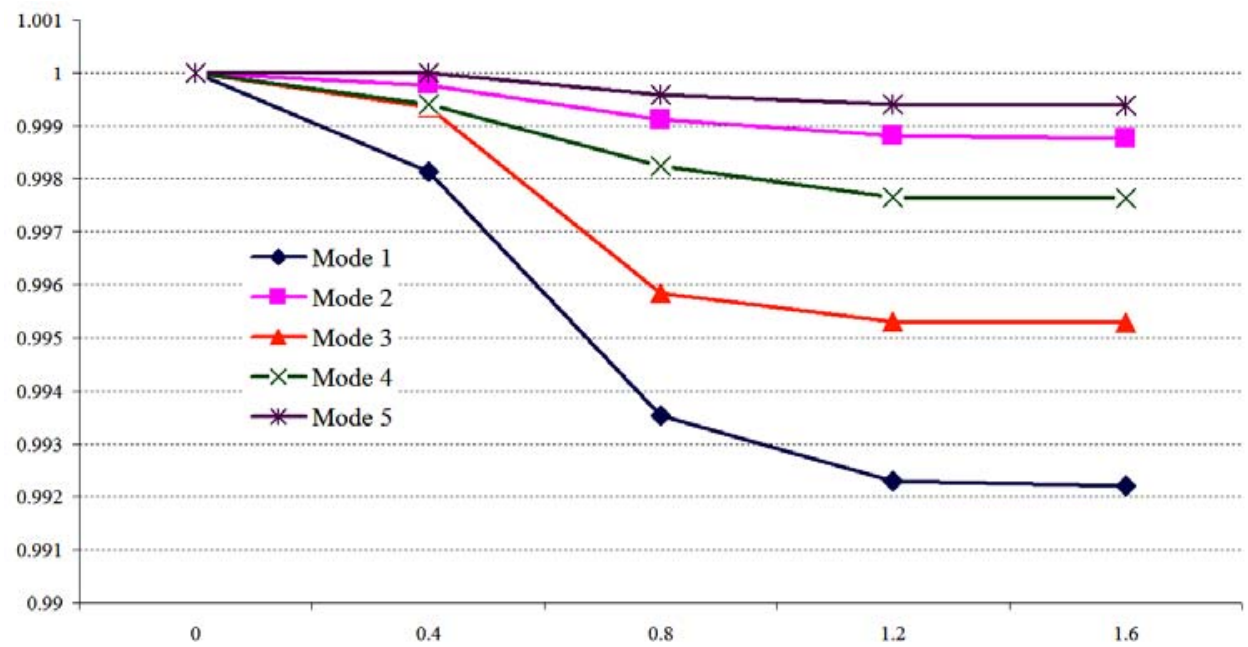

Figure 10: Achievement of frequency shift evolution from experimental tests

In the image of Fig. 10, it is clear that the evolution shapes of the frequency shift from the experimental measurements almost respect the most important aspects, as well as in the case of numerical simulation or mathematically derived. Individually taken, the vibration modes, regarding the order and the distribution of graphical evolutions, do not match a rational form, which can be assessed by comparing the mathematical, the numerical and the experimental results.

\section{CONCLUSIONS}

The analysis of beam deformed shape in the transversal vibration modes explicitly showed the presence of several areas where the displacement from the equilibrium position and the curvature/bending moment indicates maximum and null values. These areas are different in relation to vibration modes and depend on the support type. Regarding the beam characteristics length and slenderness, they do not affect the relative position of characteristic areas.

FEM applied to the multi-layered damaged beams demonstrates a deterministic relation between the areas with zero curvature, defining maximum and the variation of frequencies. Thus, the greatest values of frequency shift, due to the damage, are achieved in the areas of maximum curvature, and no frequency shift is accomplished in the zero curvature points.

The mathematical relation proposes to determine certain damage in multi-layered beams has been confirmed by FEM and an important amount of real experiments. Differences of a maximum of $3.5 \%$ were achieved by comparing results from the mathematical calculation, FEM and experimental tests.

An important accomplishment was to determine the evolution shape of frequency shift as a function of damage depth for a given damage position among the beam length. These frequency shift evolutions for isotropic materials in correspondence to the damage have been a long time ago determined by other researchers. They are available for rectangular cross sections.

In this paper, the beam loss energy dependency of its deflection modification was proven. This fact allows the establishment of a severity coefficient, as a function of the beam deflection. The 
severity coefficient, for a given vibration mode, can be computed by considering the beam deflection determined for the real damage (stiffness decreasing at exact places of the damage position).

Another advantageous method to determine the energy changing supposes that the beam deflection is achieved for the damage translated in the fixing point, and being weighted by the curvature square value for each vibration mode.

For the 5-layered beam three levels and two deep values are noticed, for which important shifts are observed, precisely at one and three mm depth. Frequency shift evolution, depending on damage depth, shows the same allure, and the amplitude is affected by the severity in that vibration mode. The reduced stiffness effect given by the middle steel layer is achieved because of the zero closing of material stress in the area.

The mathematical relation that defines frequency shift, depending on the damage depth and location, was employed to achieve a complex and complete database, generally available for any 3- or 5-layered beams. Also, based on this database a non-invasive control method has been developed. Its viability was certified by laborious laboratory tests, like those shown in this paper.

\section{Acknowledgement}

The work has been funded by the Sectoral Operational Programme Human Resources Development 2007-2013 of the Ministry of European Funds through the Financial Agreement POSDRU/159/1.5/S/132395.

\section{References}

Bilello, C. (2001). Theoretical and experimental investigation on damaged beams under moving systems, Ph.D.Thesis, Universita degli Studi di Palermo, Italy.

Caddemi, S., Calio, I. (2009). Exact closed-form solution for the vibration modes of the Euler-Bernoulli beam with multiple open cracks, Journal of Sound and Vibration 327: 473-489.

Chondros T.J., Dimarogonas A.D., Yao J. (1998). A continuous cracked beam vibration theory, Journal of Sound and Vibration 215(1): 17-34.

Doebling, S.W., Farrar, C.R., Prime, M.B. (1998). A summary review of vibration based damage identification methods, Shock Vibration Digest 30(2): 91-105.

Gillich, G.R., Abdel Wahab, M., Praisach, Z.I., Ntakpe, J.L. (2014). The influence of transversal crack geometry on the frequency changes of beams, Proceedings of International Conference on Noise and Vibration Engineering (ISMA2014) and International Conference on Uncertainty in Structural Dynamics (USD2014): 485-498.

Gillich, G.R., Birdeanu, E.D., Gillich, N., Amariei, D., Iancu, V., Jurcau, C.S. (2009). Detection of damages in simple elements, Annals of DAAAM, Proceedings of the 20th International DAAAM Symposium, vol. 20: 623-624.

Gillich, G.R., Praisach, Z., Onchis, D.M. (2010). About the effectiveness of damage detection methods based on vibration measurements, Latest trends on Engineering Mechanics, Structures, Engineering Geology, p. 204-209.

Gillich, G.R., Praisach, Z.I. (2013). Damage-patterns based method to locate discontinuities in beams, Proceedings of SPIE, vol. 8695, no. 869532, DOI: 10.1117/12.2009122.

Gillich, G.R., Praisach, Z.I. (2014). Modal identification and damage detection in beam-like structures using the power spectrum and time-frequency analysis, Signal Processing, vol. 96, Part: A, Special Issue: p. 29-44

Gillich, G.R., Praisach, Z.I., Negru, I. (2012). Damages Influence on dynamic behaviour of composite structures reinforced with continuous fibers, Materiale Plastice 49(3): 186-191. 
Liebowitz, H., Vanderveldt, H., Harris, D.W., (1967). Carrying capacity of notched column, International Journal of Solids and Structures 3(4): 489-500.

Morassi, A., Vestroni, F. (2008). Dynamic methods for damage detection in structures, CISM Courses and Lectures, Springer Wien New York, vol. 499.

Okamura, H., Liu, H.W., Chu, C.S., Liebowitz, H. (1969). A cracked column under compression, Engineering Fracture Mechanics, vol. 1, no. 3, p. 547-564.

Onchis-Moaca, D., Gillich, G.R., Frunza, R. (2012). Gradually improving the readability of the time-frequency spectra for natural frequency identification in cantilever beams, Proceedings of the 20th European Signal Processing Conference (EUSIPCO), Book Series: European Signal Processing Conference: 809-813.

Ostachowicz, W.M., Krawczuk, C. (1991). Analysis of the effect of cracks on the natural frequencies of a cantilever beam, Journal of Sound and Vibration 150(2): 191-201.

Rizos, P.F., Aspragathos, N., Dimarogonas A.D. (1990). Identification of crack location and magnitude in a cantilever beam from the vibration modes, Journal of Sound and Vibration 138(3): 381-388. 